\title{
A Rare Adult Case of Sacroiliitis due to Poststreptococcal Reactive Arthritis
}

\author{
Emre ADIGÜZEL, ${ }^{1}$ Ümmügülsüm DOĞAN DURAN, ${ }^{1}$ Yasin DEMİR, ${ }^{1}$ Sebahattin SARI, ${ }^{2}$ İsmail SAFAZ ${ }^{1}$ \\ ${ }^{1}$ Department of Physical Medicine and Rehabilitation, Gülhane Military Medical Academy, \\ Turkish Armed Forces Rehabilitation Center, Ankara, Turkey \\ ${ }^{2}$ Department of Radiology, Gülhane Military Medical Academy, Ankara, Turkey
}

\begin{abstract}
Poststreptococcal reactive arthritis is a type of sterile arthritis which occurs after a betahemolytic streptococcal throat or skin infection. It was defined as scarlatinal arthritis in 1959. Poststreptococcal reactive arthritis is a kind of non-migratory arthritis that usually affects the knee and ankle joints. Although rare, small joints and the axial skeleton may also be involved. In this report, we present a patient with unilateral sacroiliac joint inflammation and adductor enthesopathy following an upper respiratory tract infection who was eventually diagnosed with poststreptococcal reactive arthritis.

Keywords: Arthritis; reactive; sacroiliitis.
\end{abstract}

Poststreptococcal reactive arthritis (PSReA) is a type of sterile arthritis which occurs after a beta-hemolytic streptococcal throat or skin infection. ${ }^{1}$ It was first termed as scarlatinal arthritis in $1959 .{ }^{2}$ It is distinguished from acute rheumatic fever (ARF) with the absence of Jones criteria. Additionally, in PSReA the latency period is about 1-2 weeks, shorter than ARF and cardiac/kidney involvement is also rare. PSReA is a kind of nonmigratory arthritis that usually affects the knee and ankle joints. Although rare, small joints and the axial skeleton may also be involved. ${ }^{3}$ The age distribution is bimodal i.e. 8-14 and 21-37 years unlike ARF which is usually diagnosed in earlymiddle aged patients. ${ }^{4}$

In this report, we present a patient with unilateral sacroiliac joint involvement and adductor enthesopathy following an upper respiratory tract infection who was eventually diagnosed with PSReA.

\section{CASE REPORT}

A 43-year-old male patient was admitted to our hospital with severe right hip pain for the last three weeks. His complaints had first started with sore throat one month ago when he had been diagnosed as acute tonsillitis and treated with antibiotic for one week. He described that three days after the initiation of the sore throat, right hip pain had begun and that he had also suffered skin lesions at palmar sides of his both hands and plantar sides of his feet. Thereafter, he had been hospitalized to an infectious diseases clinic whereby hip magnetic resonance imaging had revealed inflammatory signal changes with edema at the right hip adductor muscle insertion. $\mathrm{He}$ had been diagnosed with septic arthritis and parenteral ceftriaxone and azithromycin had been given. As the hip pain persisted, the patient was referred to our clinic. 
On detailed questioning, he had no history of recent urinary tract infection, acute gastroenteritis, uveitis or sexually transmitted diseases. He had a sharp pain which localized on right greater trochanter extending to his posterior thigh. He reported approximately one hour of stiffness in the right hip. His body temperature was $37.2{ }^{\circ} \mathrm{C}$, pulse rate and blood pressure measurements were normal. At physical examination, active and passive right hip movements and sacroiliac compression test were painful. Physical examination was otherwise normal. Laboratory tests showed normal complete blood count with elevated erythrocyte sedimentation rate $(53 \mathrm{~mm} / \mathrm{h})$ and C-reactive protein $(8.80 \mathrm{mg} / \mathrm{L})$. Rheumatoid factor was negative. He had a positive throat culture for beta-hemolytic streptococcus which was performed at the initial hospital, but we wanted to confirm the antecedent streptococcal infection and investigated the serum antistreptolysin $\mathrm{O}$ (ASO) titer. It was $1097 \mathrm{IU} / \mathrm{mL}$ and $1806 \mathrm{IU} / \mathrm{mL}$ on admission and two weeks later, respectively. Human leukocyte antigen B27 test was positive. Liver, renal function tests and urinalysis were normal. Anti cytomegalovirus immunoglobulin M, antinuclear antibody, Venereal Disease Research Laboratory, Treponema pallidum hemagglutination assay, gonorrhea and Brucella agglutination tests were negative. Echocardiography and blood cultures were unremarkable. Plain X-rays showed normal hip joints with suspicious right sacroiliitis. Hence, we performed sacroiliac joint magnetic resonance imaging which revealed acute sacroillitis at the right sacroiliac joint (Figure 1). Accordingly, he was diagnosed as PSReA and acemetacin $60 \mathrm{mg}$ twice daily was prescribed. On the third week control visit, the patient was found to have improved significantly with decreased pain and normalized laboratory test results. Equally, ASO titer reached normal values after treatment.

\section{DISCUSSION}

Poststreptococcal arthritic syndrome which did not fulfill the diagnostic criteria for ARF was first defined as scarlatinal arthritis by Crea and Mortimer in 1959. ${ }^{2}$ Goldsmith and Long ${ }^{5}$ were the first to introduce the term PSReA for the syndrome. Initially, it was considered to be a sort of ARF whereas later on the differences between the two diseases have been established. ${ }^{6}$ Currently, PSReA is accepted to be a separate disorder from ARF by the following features: a shorter latency period between the infection and the onset of arthritis, lack of response to salicylates, lower acute phase reactants; absence of carditis, and more severe arthritis. ${ }^{7,8}$

ASO titer is not utilized for diagnosing the streptococcal infections on its own. However, it can be used to confirm the antecedent streptococcal illness. There is a close correlation between the
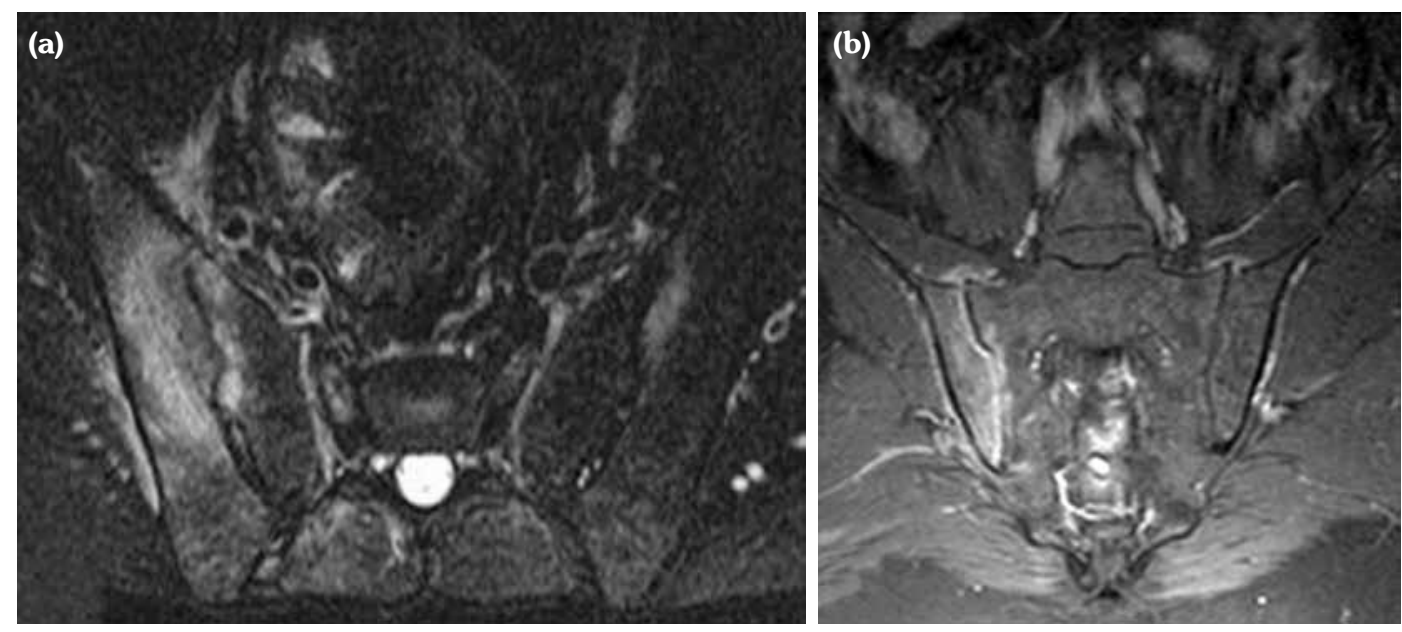

Figure 1. (a) Coronal fat saturated $T_{2}$ weighted image shows prominent $T_{2}$ signal intensity adjacent to right sacroiliac joint. There is also mild $T_{2}$ signal intensity adjacent to the upper part of the left sacroiliac joint. (b) Post-contrast fat saturated $\mathrm{T}_{1}$ images show prominent contrast enhancement in right sacroiliac joint space. There is enhancement in the juxta-articular bone marrow and soft tissues adjacent to the joint. 
serum ASO titer and sacroiliac joint involvement in PSReA. ${ }^{9}$ Therefore, ASO titer can be used to corroborate or make the differential diagnosis.

Poststreptococcal reactive arthritis is a type of non-migratory arthritis that usually affects the knee and ankle joints, and axial involvement is rare. $^{3}$ To our best notice, sacroiliitis and adductor muscle involvement has been reported in a few cases in PSReA. ${ }^{4}$ We established the diagnosis by demonstrating the unilateral sacroiliitis and the adductor enthesopathy with magnetic resonance imaging. Likewise, we would like to draw attention of physicians towards the possibility of sacroiliitis in patients with PSReA. Substantial medical history, prompt physical examination and imaging would definitely be crucial in this regard.

\section{Declaration of conflicting interests}

The authors declared no conflicts of interest with respect to the authorship and/or publication of this article.

\section{Funding}

The authors received no financial support for the research and/or authorship of this article.

\section{REFERENCES}

1. Logan D, McKee PJ. Poststreptococcal reactive arthritis. J Am Podiatr Med Assoc 2006;96:362-6.
2. Crea MA, Mortimer EA Jr. The nature of scarlatinal arthritis. Pediatrics 1959;23:879-84.

3. Ahmed S, Ayoub EM, Scornik JC, Wang CY, She JX. Poststreptococcal reactive arthritis: clinical characteristics and association with HLA-DR alleles. Arthritis Rheum 1998;41:1096-102.

4. Mackie SL, Keat A. Poststreptococcal reactive arthritis: what is it and how do we know? Rheumatology (Oxford) 2004;43:949-54.

5. Goldsmith DP, Long SS. Poststreptococcal disease of childhood: a changing syndrome. Arthritis Rheum 1982:25.

6. Guidelines for the diagnosis of rheumatic fever. Jones Criteria, 1992 update. Special Writing Group of the Committee on Rheumatic Fever, Endocarditis, and Kawasaki Disease of the Council on Cardiovascular Disease in the Young of the American Heart Association. JAMA 1992;268:2069-73.

7. Barash J, Mashiach E, Navon-Elkan P, Berkun Y, Harel L, Tauber T, et al. Differentiation of post-streptococcal reactive arthritis from acute rheumatic fever. J Pediatr 2008;153:696-9.

8. van Bemmel JM, Delgado V, Holman ER, Allaart CF, Huizinga TW, Bax JJ, et al. No increased risk of valvular heart disease in adult poststreptococcal reactive arthritis. Arthritis Rheum 2009;60:987-93.

9. Chang ST, Ku CH, Cherng SC. Evidence-based correlation between anti-streptolysin $\mathrm{O}$ serum titer and sacroiliac joint disorder. $J$ Rheumatol 2007;34:1746-52.

10. Moorthy LN, Gaur S, Peterson MG, Landa YF, Tandon M, Lehman TJ. Poststreptococcal reactive arthritis in children: a retrospective study. Clin Pediatr (Phila) 2009;48:174-82. 